Original Article

\title{
The effect of performing a dual-task on postural control and selective attention of older adults when stepping backward
}

\author{
William Wai-Nam Tsang, PhD ${ }^{1)^{*}}$, Vito Wai-Lok Chan, BSc ${ }^{1)}$, Henry Hei Wong, BSc ${ }^{1)}$, \\ TONY WAI-CheONG YIP, BSc ${ }^{1)}, \mathrm{XI}_{\mathrm{I}} \mathrm{Lu}, \mathrm{PhD}^{1,2)}$ \\ 1) Department of Rehabilitation Sciences, The Hong Kong Polytechnic University: Hung Hom, \\ Kowloon, Hong Kong, SAR, China \\ 2) Department of Rehabilitation Medicine, China-Japan Friendship Hospital, China
}

\begin{abstract}
Purpose] The purpose of the study was to investigate the postural control and cognitive performance of older adults when stepping backward with and without a concurrent cognitive task. [Subjects and Methods] Thirty young adults and twenty-eight older adults (mean age $=21.3 \pm 1.2$ and $72.2 \pm 5.7$ years, respectively) were recruited. Participants were asked to step backward and then maintain a single-leg stance for 10 seconds with and without a concurrent auditory response task. The reaction time and error rate while performing the cognitive task were recorded. Postural stability after stepping back was measured in terms of total sway path and total sway area. [Results] The older subjects had significantly longer reaction times and higher error rates in both single- and dualtasking. When dual-tasking, both groups had significantly longer reaction times than when single-tasking. Only the older adults showed significantly higher error rates. The older adults also had significantly longer total sway paths and larger total sway areas of single-leg stance after stepping back. Neither group showed a significant difference in total sway path and sway area between single- and dual-tasking. [Conclusion] Older adults have poorer cognitive performance and postural stability during both single- and dual-tasking. They tend to prioritize postural control over cognition in dual-tasking.

Key words: Dual-tasking, Postural stability, Stepping backward
\end{abstract}

(This article was submitted Apr. 17, 2016, and was accepted Jun. 16, 2016)

\section{INTRODUCTION}

Dual-tasking is common in daily life. One normally needs to maintain postural control while performing one or more other concurrent tasks such as walking while talking. An individual's attention resources and information processing capacity are presumably limited, and must be shared among all the tasks concurrently being performed ${ }^{1)}$. Therefore, when two tasks are being performed simultaneously, performance of one or both can be impaired, if together they require attention that exceeds an individual's capacity ${ }^{2}$.

Postural control, control of the body's position, orientation and balance, is often regarded as an automatic or reflexcontrolled task with minimal attentional demands. However, research has provided evidence that it requires significant attention resources ${ }^{3)}$. Performance of the postural control system declines with age due to deterioration of the visual, proprioceptive and vestibular systems, slower nerve conduction velocity and reduced musculature ${ }^{4)}$. Studies have shown that the attentional demands of balance control increase with age in compensation, even as general attention resources decrease ${ }^{5,6)}$. Consequently, older adults are more likely to have insufficient attention resources for effective postural control in dual-task situations, which would affect their dual-task performance. Bergamin et al. reported that older adults have significantly higher

\footnotetext{
*Corresponding author. William Wai-Nam Tsang (E-mail: william.tsang@polyu.edu.hk)

(C)2016 The Society of Physical Therapy Science. Published by IPEC Inc.

This is an open-access article distributed under the terms of the Creative Commons Attribution Non-Commercial No Derivatives (by-nc-nd) License $<$ http://creativecommons.org/licenses/by-nc-nd/4.0/>.
} 
values of sway area and center of pressure velocity in older adults during dual-tasks when compared with young adults ${ }^{7)}$. This suggests why the risk of balance loss, and hence falling, increases among older adults ${ }^{8}$. Older adults' dual-task performance may also be influenced by other factors, such as task difficulty, postural threat, stimulus modalities or response modes. In tasks with increasing difficulty, such as walking backward, an increased cognitive demand occurs and the concurrent execution of a cognitive task may be compromised ${ }^{9}$.

Falls are a common problem for the aged. Among community-dwelling adults aged 65 or above, approximately $30 \%$ of them experience a fall at least once each year ${ }^{10)}$. Impact on the hip is likely in a backward or sideways fall ${ }^{11)}$, and direct impact on the hip in a fall from standing height can easily be forceful enough to cause a hip fracture in older adults ${ }^{12}$. Besides physical impact, other possible consequences include psychological problems, a reduction in subsequent activity or even social isolation due to fear of falling ${ }^{13)}$.

Older people often have difficulty stepping backward. Many falls occur during transitional movements which require an older person to step backward. Backward walking is associated with reduced velocity and stride length, and increased gait variability, and these parameters are associated with a greater fall risk in older adults ${ }^{14)}$. Examples include pulling open a door, backing to sit on a chair, or responding to a forward perturbation. These are common situations that older adults encounter in daily life, but the effects of aging on postural control when stepping backward have not been well-studied. Therefore, this study was designed to examine (i) whether there are differences between young and older adults in cognitive performance and postural control after stepping back, and (ii) to what extent dual-tasking affects cognitive performance and postural control. The hypothesis was that older adults would have poorer cognitive performance and postural stability after stepping back, especially while dual-tasking.

\section{SUBJECTS AND METHODS}

A total of fifty-eight subjects, including thirty young adults and twenty-eight older adults, participated in this cross-sectional study. The young adults were students attending the Hong Kong Polytechnic University. The older subjects, communitydwelling adults aged 65 or above, were recruited from elderly centers in Hong Kong. The subjects were all independent in their daily living activities, able to walk unaided, and native Cantonese speakers. Candidates were excluded if they had a neurological disorder, myocardial infarction or heart failure, uncontrolled diabetes or hypertension, deformity of the lower limbs, a history of musculoskeletal injury to either lower limb in the previous 12 months, or cognitive or hearing impairment. The validated Cantonese version of the Mini-Mental State Examination was used to screen the older subjects for cognitive impairment ${ }^{15}$. Candidates with scores less than 24 were regarded as impaired and excluded from the study ${ }^{16)}$. Subjects who reported dizziness on the day of the assessment or failed to follow the verbal commands were also excluded. Ethics approval was obtained from the Ethics Committee of the Hong Kong Polytechnic University. Written informed consent was obtained from all of the participants prior to their participation.

Each participant performed three tasks: an auditory Stroop test and a stepping-backward task with and without a concurrent auditory Stroop test. The order of the three tasks was randomized by drawing lots. The classic Stroop test requires subjects to name the color in which a word is printed where the word itself is the name of a different color. Subjects react more quickly and accurately to congruent stimuli (when the word corresponds to the color of the printing) than to incongruent stimuli (where the word and the print color differ). The test has been shown to demand selective attention ${ }^{17)}$. An auditory version of the test was employed in this study. Cantonese is a tonal language where meaning depends both on the phoneme and the tone in which it is pronounced. Two Cantonese words meaning "high" and "low" were used in this test, and they were pronounced at either a high or low pitch, generating four combinations of pitch and meaning. The subjects were required to respond to the voice as quickly as possible by pressing the right thumb switch when a high pitch voice was heard or pressing the left one for low pitch, disregarding the meaning of the word as pronounced. For the test, each subject was seated in a quiet room. Each participant had four familiarization trials (one for each combination) before sixteen test trials (four trials per combination in a random order). The reaction time (the time between the sound and the moment when the switch was pressed) and error rate (percentage of wrong responses) were recorded.

The stepping-backward task started with the subject standing barefoot on a force plate (Model OR6-5-1000, Advanced Mechanical Technologies Inc., Newton, MA, USA) with the feet shoulder-width apart, looking at a fixed visual target placed two meters from the center of the force plate. The visual fixation during the task served as gaze stabilization. The participants were required to make a step backward from one force plate onto another in response to a verbal command. The dominant leg, which was defined as the leg used to kick a ball, was used to step back ${ }^{18}$. Meanwhile, before landing, the subject was required to press one of the thumb switches (without an auditory Stroop test). This served to control for any possible perturbation of postural control arising from the action of pressing a thumb switch. After stepping back the subject was required to maintain a single-leg stance on the dominant leg for 10 seconds and keep looking at the fixed visual target. A prolonged phase of double-leg stance (with the non-dominant leg stays in the front force plate) or a shortened single-leg stance of the dominant leg occurs when postural control deteriorates. Thus, the duration of the double-leg stance and the sway parameters during the single-leg stance were recorded and compared between the older and young participants. A familiarization trial was given before the four test trials, and rests were allowed if necessary. The center of pressure (COP) data collected during the first 5 seconds of the single-leg stance were analyzed to quantify postural control, since the average single-leg stance duration 
of older adults is normally about 6 to 7 seconds ${ }^{19}$ ). Two body-sway measures: (i) the total sway path of the COP and (ii) the sway area covered by the maximum sway excursion, were used to evaluate the postural control performance after stepping back. The data were recorded at a sampling frequency of $1,000 \mathrm{~Hz}$ and then re-sampled at $120 \mathrm{~Hz}$ for analysis using Matlab 7.1 software (The Math-Works Inc., Natick, USA).

When performing stepping-backward with a concurrent cognitive task, subjects were requested to respond to the auditory Stroop test as quickly and accurately as possible while simultaneously performing the stepping-backward task. The auditory Stroop test was triggered either when the subjects lifted their dominant leg (detected by a pressure sensor placed underneath their heels), or stepped back onto the force plate. They were instructed that the auditory Stroop test could be triggered at any time during stepping back or the single-leg stance. Familiarization trials were conducted before each subject performed the eight test trials.

Version 20.0 of the commercially available Statistical Package for the Social Sciences (SPSS) software (IBM Corp., Armonk, NY, USA) was used for statistical analysis. The mean and standard deviation were used to characterize continuous data. The average ages, heights and weights of the young and older subjects were compared using the independent $\mathrm{t}$-test. The $\chi^{2}$ test was used to compare the gender distribution of the two groups. Repeated measures multivariate analysis of variance (MANOVA) was used to compare the results of the auditory Stroop tests, the stepping-back task and the dual-task performances between the two groups. Univariate tests were conducted for each of the measures when an overall statistically significant difference was found. The confidence level for statistical significance was chosen as 0.05 .

\section{RESULTS}

Thirty younger and twenty-eight older adults participated in this study. The baseline characteristics of the two groups are shown in Table 1 . The two groups differed significantly in terms of age and height (both $\mathrm{p}<0.001$ ), but there was no significant difference in their gender distribution or weights.

A significant overall (group $\times$ task) difference $(\mathrm{F}=24.835 ; \mathrm{p}<0.001)$ in the auditory Stroop test was found by repeated measures MANOVA. Univariate analysis demonstrated a significant (group $\times$ task) difference in reaction times $(\mathrm{p}<0.001)$, but not in error rates $(\mathrm{p}=0.064)$ (Table 2$)$. The independent $\mathrm{t}$-test showed there were significant between-group differences in average reaction times and error rates of both the single- and dual-tasking $(\mathrm{p}<0.001)$. The paired t-test showed that, when comparing dual-tasking with single-tasking, both the young and older subjects had significantly increased average reaction times ( $p=0.002$ and $p<0.001$, respectively), but that the older adults had significantly higher average error rates $(p=0.001)$ but this was not evident among the young adults $(\mathrm{p}=0.1)$.

Since the average height of the two groups was significantly different, height was treated as a covariate in the statistical analysis. In addition, the double-leg stance durations from the dominant leg landing to the lifting of the non-dominant leg were significantly longer for the older subjects in both single- and dual-tasking (both $\mathrm{p}<0.001$ ) (Table 3 ). The younger subjects were simply nimbler and that would be expected to affect subsequent postural performance. Therefore, the double-leg stance durations were also treated as covariates in the statistical analysis. Repeated measures MANOVA showed no significant group $\times$ task difference in either average path length or the area subtended by COP peregrination during the first 5 seconds of the single-leg stance $(\mathrm{p}>0.05)$. The independent t-test revealed there were significant between-group differences in terms

Table 1. Participants characteristics

\begin{tabular}{lcc}
\hline Variables & Young adult group $(\mathrm{n}=30)$ & Older adult group $(\mathrm{n}=28)$ \\
\hline Age $($ years $)$ & $21.3 \pm 1.2$ & $72.2 \pm 5.7^{*}$ \\
Height $(\mathrm{cm})$ & $163.9 \pm 7.3$ & $154.8 \pm 6.4^{*}$ \\
Weight $(\mathrm{kg})$ & $54.8 \pm 8.8$ & $59.1 \pm 8.6$ \\
Female, $\mathrm{n}(\%)$ & $22(73.3)$ & $21(75.0)$ \\
\hline
\end{tabular}

Mean \pm standard deviation. *Significant difference between the young and older adults groups.

Table 2. Auditory Stroop test results during single- and dual-tasking

\begin{tabular}{lcccccc}
\hline & \multicolumn{3}{c}{ Young adult group $(\mathrm{n}=30)$} & \multicolumn{3}{c}{ Older adult group $(\mathrm{n}=28)$} \\
\hline & Single task & Dual task & $\%$ change $(\%)$ & Single task & Dual task & $\%$ change $(\%)$ \\
\hline Reaction time (s) & $0.62 \pm 0.10$ & $0.69 \pm 0.15$ & $+11^{* *}$ & $1.03 \pm 0.32^{*}$ & $1.81 \pm 0.46^{*}$ & $+76^{* *}$ \\
Error rate (\%) & $4.6 \pm 5.3$ & $8.9 \pm 12.0$ & +95 & $15.2 \pm 11.3^{*}$ & $27.2 \pm 21.0^{*}$ & $+80^{* *}$ \\
\hline
\end{tabular}

Mean \pm standard deviation.

*Significant difference between the young and older adults groups.

**Significant difference between single- and dual-tasking. 
of both total sway path and total sway area for both the single- and dual-tasking. But the paired t-test showed that neither the young nor the older adults had a significant difference in total sway path of the COP or the sway area in the comparison of single- versus dual-tasking (all $\mathrm{p}>0.05$ ).

\section{DISCUSSION}

This study investigated the postural control and cognitive performance of older adults in both single- and dual-tasking. Compared with young adults, older adults showed significantly longer reaction times, higher error rates, longer total sway paths and larger total sway areas when stepping backward with and without a concurrent cognitive task. When dual-tasking, both groups had significantly longer reaction times than when single-tasking, but only older adults showed significantly higher error rates. These results suggest that older adults tend to prioritize postural control over cognition when performing a dual-task.

For cognitive performance, the results showed that the older adults had a significantly longer average reaction time, and a higher error rate in the auditory Stroop test than the young adults. This was as expected because of the well-understood decline with age in the central processing capacity of the brain ${ }^{20)}$. When an additional postural task was included, both groups demonstrated increased average reaction times. It was hypothesized that if two tasks were performed simultaneously and exceeded the processing capacity, the performance of one or both tasks would be degraded ${ }^{2}$. The competition for attention resources generated in this experimental protocol was sufficient that the cognitive performance of both groups was affected in the dual-tasking condition.

These results are comparable to those of a study published by our research group in which the participants stepped down from a $19-\mathrm{cm}$ platform with and without an accompanying auditory Stroop test ${ }^{18)}$. While the cognitive task was the same, the postural task was different from the present study. Nevertheless, those results showed that older adults had significantly longer reaction times and higher error rates in dual-tasking than single-tasking, as well as when compared with young adults in both conditions. However, the present study found young adults had slower reaction times in dual-tasking than in single-tasking. This was probably due to the task sequence randomization adopted in the present study, which might have minimized the practice effect on the cognitive task results. Related to this, Lajoie and co-workers examined the effect on auditory reaction time of the maintenance of static posture and gait using eight young and eight older adults. They also found that the reaction times of both groups were fastest during sitting, and concluded that both young and older adults required more cognitive resources for standing and walking than for sitting ${ }^{21)}$. Bergamin and colleagues ${ }^{7)}$ investigated the effects of different secondary tasks, namely a Brooks spatial-memory task (visual construct), counting backwards aloud test (verbal), and mental arithmetic task (cognitive) on a static postural stability task. They found that the verbal task influenced the performance of the postural task most and they ascribed this finding to either vocalization and/or cognitive demands. The adoption of the auditory Stroop test in this study, with the pressing of the thumb switch arbitrarily during stepping backward (single-task), eliminated the influence of a secondary physical task on the postural control task. Such a protocol could confine the secondary task to its cognitive demand.

Compared with the younger subjects, the older ones showed significantly longer average sway paths and larger average sway areas in both single- and dual-tasking. This result is in agreement with our previous study on stepping down, which found that older adults have larger body sway than young adults in both single and dual tasks ${ }^{18)}$. Possible reasons for the increased postural sway include the age-related decline in the speed of torque development in the lower extremities ${ }^{22}$, delayed onset of postural muscle activation ${ }^{23}$, and a diminished capacity to detect and integrate somatosensory information ${ }^{24)}$. All of these may contribute to a greater sway amplitude and a larger sway area. In contrast, the younger subjects' faster ankle torque development and quicker postural muscle responses should have helped them restore balance more quickly, leading to better postural performance ${ }^{25}$.

The older subjects had similar performance in terms of both postural control measures, but their cognitive performance declined significantly in dual-tasking when compared with single-tasking, showing that they were prioritizing postural control. This accords with the "posture first" hypothesis suggested by Shumway-Cook which proposes that older adults allocate resources to postural control at the expense of cognitive performance in dual-tasking conditions ${ }^{26)}$. It indicates that the motor

Table 3. Center of pressure excursions during single- and dual-tasking

\begin{tabular}{lcccccc}
\hline & \multicolumn{3}{c}{ Young adult group $(\mathrm{n}=30)$} & \multicolumn{3}{c}{ Older adult group $(\mathrm{n}=28)$} \\
\hline & Single task & Dual task & $\%$ change $(\%)$ & Single task & Dual task & $\%$ change $(\%)$ \\
\hline COP path $(\mathrm{mm})$ & $92.3 \pm 66.1$ & $101.8 \pm 71.0$ & +10 & $273.1 \pm 54.2^{*}$ & $286.0 \pm 60.5^{*}$ & +5 \\
COP area $\left(\mathrm{mm}^{2}\right)$ & $474.8 \pm 343.8$ & $679.5 \pm 565.5$ & +43 & $1,081.5 \pm 444.9^{*}$ & $1,180.0 \pm 574.2^{*}$ & +9 \\
Double-leg stance duration $\dagger(\mathrm{ms})$ & $444.5 \pm 125.4$ & $459.4 \pm 108.2$ & +3 & $771.1 \pm 287.4^{*}$ & $705.2 \pm 201.4^{*}$ & -9 \\
\hline
\end{tabular}

Mean \pm standard deviation.

*Significant difference between the young and older adults groups.

$\dagger$ For co-variate analysis. 
task is prioritized over cognition in older adults. Task prioritization tends to be more obvious when the motor task involves a risk of falling, especially for frail older people with balance impairments for whom a fall could have critical survival significance ${ }^{9)}$.

Backward stepping may be considered as an alternative to stepping down, which is presumably a more challenging postural task ${ }^{18)}$, for assessing the balance ability of older adults and/or identifying those at greater risk of falling. The postural task may be more suitable for frail subjects, such as patients with Parkinson disease, who often have backward walking deficits which might lead to backward falls. It has also been suggested that assessing backward walking may be illustrative of the degree of basal ganglia impairment ${ }^{27)}$. However, more balance-related data should be collected before a cut-off point for differentiation can be definitively set. The dual-task paradigm is more demanding of attention, so it might be a more sensitive test than the single-task protocol for testing postural control ${ }^{28)}$.

Since this was a cross-sectional study, no causality can be attributed. Also, most of the young subjects were university students. The generalizability of the results of the cognitive assessments might be affected due to this limited variety in educational level.

Only the auditory Stroop test was used in this study. It is plausible that different cognitive demands will influence postural control. Further studies are needed to examine the motor and cognitive costs when different cognitive tasks are performed during stepping backward.

No electromyographic data was collected, so the muscle recruitment strategies adopted by subjects during stepping back remain to be explored. This could be included in future investigations to search for differences in the strategies employed by different subject groups. Future studies might fruitfully compare older fallers with similar non-fallers to find out if there is any difference in postural control performance between them during single- and dual-task activities.

It has been suggested that exercise training is beneficial for the static and dynamic balance of older adults with multiple disease conditions performing single and dual tasks ${ }^{29}$. However, insufficient evidence has been found to verify these benefits in healthy older adults ${ }^{30)}$. Therefore, one possible direction for future study is to evaluate intervention trials which may increase the performance of postural control and selective attention while walking backward. High quality studies should compare different exercise modalities, and study the optimal dose of exercise needed to improve dual-task performance and reduce the risk of fall.

In conclusion, young adults had better postural control and superior cognitive performance in both single- and dualtasking as they showed significantly shorter reaction times and lower error rates in the auditory Stroop test, as well as shorter total sway paths and smaller total sway areas in the postural task. Although neither group showed a significant difference in postural control between single- and dual-tasking, they had significantly longer reaction times during dual-tasking, indicating older adults tend to prioritize postural control over cognitive performance when dual-tasking.

\section{ACKNOWLEDGEMENTS}

The authors thank the subjects for their participation. Thanks are also due to Mr. Bill Purves for his English editorial advice. This project received no financial support, and there is no potential conflicts of interest.

\section{REFERENCES}

1) Kerr B, Condon SM, McDonald LA: Cognitive spatial processing and the regulation of posture. J Exp Psychol Hum Percept Perform, 1985, 11: 617-622. [Medline] [CrossRef]

2) Dault MC, Frank JS, Allard F: Influence of a visuo-spatial, verbal and central executive working memory task on postural control. Gait Posture, 2001, 14: 110-116. [Medline] [CrossRef]

3) Woollacott M, Shumway-Cook A: Attention and the control of posture and gait: a review of an emerging area of research. Gait Posture, 2002, 16: 1-14. [Medline] [CrossRef]

4) Maylor EA, Wing AM: Age differences in postural stability are increased by additional cognitive demands. J Gerontol B Psychol Sci Soc Sci, 1996, 51: 143-154. [Medline] [CrossRef]

5) Brown LA, Shumway-Cook A, Woollacott MH: Attentional demands and postural recovery: the effects of aging. J Gerontol A Biol Sci Med Sci, 1999, 54: M165-M171. [Medline] [CrossRef]

6) Johnson MK, Mitchell KJ, Raye CL, et al.: An age-related deficit in prefrontal cortical function associated with refreshing information. Psychol Sci, 2004, 15: 127-132. [Medline] [CrossRef]

7) Bergamin M, Gobbo S, Zanotto T, et al.: Influence of age on postural sway during different dual-task conditions. Front Aging Neurosci, 2014, 6: 271. [Medline] [CrossRef]

8) Teasdale N, Simoneau M: Attentional demands for postural control: the effects of aging and sensory reintegration. Gait Posture, 2001, 14: 203-210. [Medline] [CrossRef]

9) Schaefer S: The ecological approach to cognitive-motor dual-tasking: findings on the effects of expertise and age. Front Psychol, 2014, 5: 1167. [Medline] [CrossRef]

10) Gill T, Taylor AW, Pengelly A: A population-based survey of factors relating to the prevalence of falls in older people. Gerontology, 2005, 51: 340-345. [Med- 
line] [CrossRef]

11) Hsiao ET, Robinovitch SN: Common protective movements govern unexpected falls from standing height. J Biomech, 1998, 31: 1-9. [Medline] [CrossRef]

12) Robinovitch SN, Hayes WC, McMahon TA: Prediction of femoral impact forces in falls on the hip. J Biomech Eng, 1991, 113: 366-374. [Medline] [CrossRef]

13) Zettel JL, McIlroy WE, Maki BE: Effect of competing attentional demands on perturbation-evoked stepping reactions and associated gaze behavior in young and older adults. J Gerontol A Biol Sci Med Sci, 2008, 63: 1370-1379. [Medline] [CrossRef]

14) Fritz NE, Worstell AM, Kloos AD, et al.: Backward walking measures are sensitive to age-related changes in mobility and balance. Gait Posture, 2013, 37: 593-597. [Medline] [CrossRef]

15) Chiu HF, Lee HC, Chung WS, et al.: Reliability and validity of the Cantonese version of the Mini-Mental State Examination: a preliminary study. J Hong Kong Coll Psychiatrists, 1994, 4: 25-28.

16) Folstein M, Anthony JC, Parhad I, et al.: The meaning of cognitive impairment in the elderly. J Am Geriatr Soc, 1985, 33: 228-235. [Medline] [CrossRef]

17) Lezak M: Neuropsychological Assessment, 3rd ed. New York: Oxford University Press, 1995.

18) Tsang WW, Lam NK, Lau KN, et al.: The effects of aging on postural control and selective attention when stepping down while performing a concurrent auditory response task. Eur J Appl Physiol, 2013, 113: 3021-3026. [Medline] [CrossRef]

19) Li F, Harmer P, Fisher KJ, et al.: Tai Chi and fall reductions in older adults: a randomized controlled trial. J Gerontol A Biol Sci Med Sci, 2005, 60: 187-194. [Medline] [CrossRef]

20) Verhaeghen P, Steitz DW, Sliwinski MJ, et al.: Aging and dual-task performance: a meta-analysis. Psychol Aging, 2003, 18: 443-460. [Medline] [CrossRef]

21) Lajoie Y, Teasdale N, Bard C, et al.: Upright standing and gait: are there changes in attentional requirements related to normal aging? Exp Aging Res, 1996, 22: 185-198. [Medline] [CrossRef]

22) Bento PC, Pereira G, Ugrinowitsch C, et al.: Peak torque and rate of torque development in elderly with and without fall history. Clin Biomech (Bristol, Avon), 2010, 25: 450-454. [Medline] [CrossRef]

23) Laughton CA, Slavin M, Katdare K, et al.: Aging, muscle activity, and balance control: physiologic changes associated with balance impairment. Gait Posture, 2003, 18: 101-108. [Medline] [CrossRef]

24) Qiu F, Cole MH, Davids KW, et al.: Enhanced somatosensory information decreases postural sway in older people. Gait Posture, 2012, 35: 630-635. [Medline] [CrossRef]

25) Lin SI, Woollacott MH: Postural muscle responses following changing balance threats in young, stable older, and unstable older adults. J Mot Behav, 2002, 34 37-44. [Medline] [CrossRef]

26) Shumway-Cook A, Woollacott M, Kerns KA, et al.: The effects of two types of cognitive tasks on postural stability in older adults with and without a history of falls. J Gerontol A Biol Sci Med Sci, 1997, 52: M232-M240. [Medline] [CrossRef]

27) Hackney ME, Earhart GM: Backward walking in Parkinson's disease. Mov Disord, 2009, 24: 218-223. [Medline] [CrossRef]

28) Toulotte $\mathrm{C}$, Thevenon A, Watelain E, et al.: Identification of healthy elderly fallers and non-fallers by gait analysis under dual-task conditions. Clin Rehabil, 2006, 20: 269-276. [Medline] [CrossRef]

29) Zanotto T, Bergamin M, Roman F, et al.: Effect of exercise on dual-task and balance on elderly in multiple disease conditions. Curr Aging Sci, 2014, 7: 115-136. [Medline] [CrossRef]

30) Gobbo S, Bergamin M, Sieverdes JC, et al.: Effects of exercise on dual-task ability and balance in older adults: a systematic review. Arch Gerontol Geriatr, 2014, 58: 177-187. [Medline] [CrossRef] 